\title{
Hecke correspondences for Hilbert schemes of reducible locally planar curves
}

\author{
Oscar Kivinen
}

\begin{abstract}
Let $C$ be a complex, reduced, locally planar curve. We extend the results of Rennemo to reducible curves by constructing an algebra $A$ acting on $V=\bigoplus_{n \geqslant 0} H_{*}^{\mathrm{BM}}\left(C^{[n]}, \mathbb{Q}\right)$, where $C^{[n]}$ is the Hilbert scheme of $n$ points on $C$. If $m$ is the number of irreducible components of $C$, we realize $A$ as a subalgebra of the Weyl algebra of $\mathbb{A}^{2 m}$. We also compute the representation $V$ in the simplest reducible example of a node.
\end{abstract}

\section{Introduction}

Let $C$ be a complex, reduced, locally planar curve. We are interested in studying the homologies of the Hilbert schemes of points $C^{[n]}$. In the case when $C$ is integral, work of Rennemo, Migliorini-Shende, and Maulik-Yun [Ren18, MS13, MY14] relates these homologies to the homology of the compactified Jacobian of $C$ equipped with the perverse filtration. Furthermore, work of Migliorini-Shende-Viviani [MSV18] considers an extension of these results to reduced but possibly reducible curves.

Following Rennemo, we approach the problem of computing the homologies of the Hilbert schemes in question from the point of view of representation theory. In [Ren18], a Weyl algebra in two variables acting on $V:=\bigoplus_{n \geqslant 0} H_{*}^{\mathrm{BM}}\left(C^{[n]}\right)$ was constructed for integral locally planar curves, and $V$ was described in terms of the representation theory of the Weyl algebra. The superscript BM denotes Borel-Moore homology. When $C$ has $m$ irreducible components, we construct an algebra $A$ acting on $V$, where $A$ is an explicit subalgebra of the Weyl algebra in $2 m$ variables. The main result is the following.

TheOrem 1.1. If $C=\bigcup_{i=1}^{m} C_{i}$ is a decomposition of $C$ into irreducible components, the space $V=\bigoplus_{n \geqslant 0} H_{*}\left(C^{[n]}, \mathbb{Q}\right)$ carries a bigraded action of the algebra

$$
A=A_{m}:=\mathbb{Q}\left[x_{1}, \ldots, x_{m}, \partial_{y_{1}}, \ldots, \partial_{y_{m}}, \sum_{i=1}^{m} y_{i}, \sum_{i=1}^{m} \partial_{x_{i}}\right],
$$

where $V=\bigoplus_{n, d \geqslant 0} V_{n, d}$ is graded by the number of points $n$ and homological degree $d$. Moreover, the operators $x_{i}$ have degree $(1,0)$, and the operators $\partial_{y_{i}}$ have degree $(-1,-2)$ in this bigrading. In effect, the operator $\sum y_{i}$ has degree $(1,2)$, and the operator $\sum \partial_{x_{i}}$ has degree $(-1,0)$.

Received 05 December 2017, accepted in final form 05 September 2018.

2010 Mathematics Subject Classification 14C05.

Keywords: Hilbert scheme of points, representation theory.

This journal is (C) Foundation Compositio Mathematica 2019. This article is distributed with Open Access under the terms of the Creative Commons Attribution Non-Commercial License, which permits non-commercial reuse, distribution, and reproduction in any medium, provided that the original work is properly cited. For commercial re-use, please contact the Foundation Compositio Mathematica.

This work was partially funded by the Vilho, Yrjö and Kalle Visälä Foundation of the Finnish Academy of Science and Letters, a Fulbright Graduate Grant, and NSF grants DMS-1700814 and DMS-15593381. 


\section{Homology of Hilbert SCHEMES OF PLANAR CURVES}

Remark 1.2. The algebra $A$ does not depend on $C$ but only on the number of components $m$.

Remark 1.3. An argument similar to [Ren18, Theorem 1.2] shows that $V$ is free over $\mathbb{Q}\left[x_{i}\right]$ for any $i=1, \ldots, m$ and also over $\mathbb{Q}\left[\sum_{i=1}^{m} y_{i}\right]$. Through the Oblomkov-Rasmussen-Shende (ORS) conjectures (see below), this may be seen as a version of Rasmussen's remark in [Ras15] that the triply graded homology of the link $L$ of $C$ is free over the homology of an unlink corresponding to a component of $L$.

In Section 2, we will discuss the relevant geometry, namely the deformation theory of locally planar curves. In particular, we prove that the relative families of (flag) Hilbert schemes have smooth total spaces, which is crucial for applying a bivariant homology formalism, described in Section 3.1. We then define the action of the generators of $A$ on $V$ by explicit geometric constructions in Section 3 and prove the commutation relations in Section 4. In Section 5, we describe the representation $V$ of the algebra $A_{2}$ in the example of the node. More precisely, we have the following result.

Theorem 1.4. When $C=\left\{x^{2}=y^{2}\right\} \subset \mathbb{P}^{2}$, we have

$$
V \cong \frac{\mathbb{Q}\left[x_{1}, x_{2}, y_{1}, y_{2}\right]}{\mathbb{C}\left[x_{1}, x_{2}, y_{1}+y_{2}\right]\left(x_{1}-x_{2}\right)}
$$

as an $A$-module, where

$$
A=\mathbb{Q}\left[x_{1}, x_{2}, \partial_{x_{1}}+\partial_{x_{2}}, y_{1}+y_{2}, \partial_{y_{1}}, \partial_{y_{2}}\right] \subset \operatorname{Weyl}\left(\mathbb{A}^{4}\right) .
$$

Remark 1.5. Although seeing the algebra $A$ for the first time immediately raises the question whether we can define the operators $\partial_{x_{i}}$ or multiplication by $y_{i}$ separately, that is, extend this action to the whole Weyl algebra, this example shows that it is in fact not possible to do this while retaining the module structure for $V$.

Locally planar curve singularities are connected naturally to topics ranging from the Hitchin fibration [Ngô06] to HOMFLY-PT homology of the links of the singularities [ORS18, GORS14]. For example, from [ORS18] we have the following.

Conjecture 1.6. If $C$ has a unique singularity at 0 , its link is by definition the intersection of $C$ with a small three-sphere around 0 . There is an isomorphism

$$
V_{0}^{c} \cong \mathrm{HHH}_{a=0}(\text { Link of } C),
$$

where $V_{0}^{c}=\bigoplus_{n \geqslant 0} H^{*}\left(C_{0}^{[n]}\right)$ is the cohomology of the punctual Hilbert scheme and $\mathrm{HHH}(-)$ is the triply graded HOMFLY-PT homology of Khovanov and Rozansky [KR08].

This conjecture is still wide open. Recently, advances on the knot homology side have been made by Hogancamp, Elias and Mellit [EH16, Hog17, Mel16], who compute the HOMFLY-PT homologies of, for example, $(n, n)$-torus links using algebraic techniques. As the $(n, n)$-torus links appear as the links of the curves $C=\left\{x^{n}=y^{n}\right\} \subset \mathbb{P}^{2}$, a partial motivation for this work was to study the Hilbert schemes of points on these curves.

Remark 1.7. There are many natural algebras acting on $\mathrm{HHH}(-)$, for example the positive half of the Witt algebra, as proven in [KR16]. It might be possible that the actions of the operators $\mu_{+}=\sum_{i} \partial_{x_{i}}$ and $x_{i}$ on $V$ are related to this action.

In the case when $C=\left\{x^{p}=y^{q}\right\}$ for coprime $p$ and $q$, there is an action of the spherical rational Cherednik algebra of $\mathrm{SL}_{n}$ with parameter $c=p / q$ on the cohomology of the compactified Jacobian 


\section{O. Kivinen}

of $C$ [VV09, OY16], or rather its associated graded with respect to the perverse filtration, which is intimately related to the space $V$. For arbitrary torus links, it might still be true that $V$ or its variants carry some form of an action of a rational Cherednik algebra.

\section{Geometry of Hilbert schemes of points}

We describe the general setup for this paper. Fix a locally planar reduced curve $C / \mathbb{C}$, and let $C=\bigcup_{i=1}^{m} C_{i}$ be a decomposition of $C$ to irreducible components. We will be working with versal deformations of $C$.

Definition 2.1. If $X$ is a projective scheme, a versal deformation of $X$ is a map of germs $\pi: \mathcal{X} \rightarrow B$ such that $B$ is smooth, $\pi^{-1}(0)=X$, and given $\pi^{\prime}: \mathcal{X}^{\prime} \rightarrow B^{\prime}$ with $\pi^{\prime-1}\left(b^{\prime}\right)=X$ there exists a $\phi: B^{\prime} \rightarrow B$ such that $b^{\prime}$ maps to 0 and $\pi^{\prime}$ is the pullback of $\pi$ along $\phi$. If $T_{0} B$ coincides with the first-order deformations of $X$ or, in other words, the base $B$ is of minimal dimension, we call $\pi$ a miniversal deformation.

We call a family of locally planar, reduced, complex algebraic curves over a smooth base $B$ locally versal at $b \in B$ if the induced deformations of the germs of the singular points of $\pi^{-1}(b)$ are versal. We are interested in the smoothness of relative families of Hilbert schemes of points for such deformations, needed for example for Lemma 4.3.

Definition 2.2. If $\mathcal{X} \rightarrow B$ is any family of projective schemes and $P(t)$ is any Hilbert polynomial, we denote the relative Hilbert scheme of this family by $\mathcal{X}^{P(t)}$. By definition, Hilbert schemes are defined for families [Gro95], and we note here that at closed points $b \in B$, the fibers of the relative Hilbert scheme are exactly $\operatorname{Hilb}^{P(t)}\left(\mathcal{X}_{b}\right)$.

We now consider the tangent spaces to (relative) Hilbert schemes.

Lemma 2.3. For any projective scheme $X$ and a flag of subschemes $X_{1} \subset \cdots \subset X_{k}$ in $X$ with fixed Hilbert polynomials $P_{1}(t), \ldots, P_{k}(t)$, the Zariski tangent space is given by

$$
T_{\left(X_{1}, \ldots, X_{n}\right)} \operatorname{Hilb}^{\overrightarrow{P(t)}}(X) \cong H^{0}\left(X, \mathcal{N}_{\left(X_{1}, \ldots, X_{m}\right) / X}\right)
$$

where the sections of the normal sheaf $\mathcal{N}_{\left(X_{1}, \ldots, X_{m}\right) / X} \subseteq \bigoplus_{i=1}^{k} \mathcal{N}_{X_{i} / X}$ are tuples $\left(\xi_{1}, \ldots, \xi_{k}\right)$ of normal vector fields such that $\left.\xi_{i}\right|_{X_{j}}=\xi_{j}$ modulo $\mathcal{N}_{X_{j} / X_{i}}$ whenever $X_{i} \supseteq X_{j}$. The normal sheaf is, by definition, the sheaf of germs of commutative diagrams of homomorphisms of $\mathcal{O}_{X}$-modules of the form

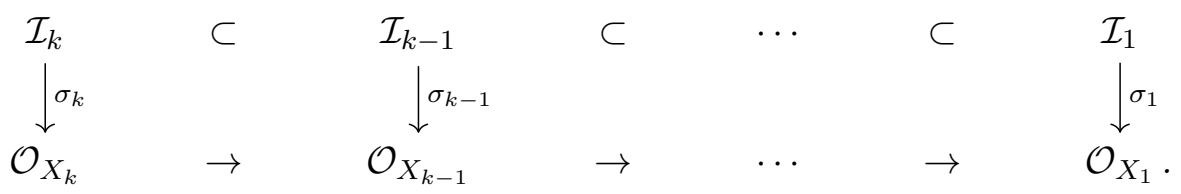

Proof. Note that from first-order deformation theory, it immediately follows that if $k=1$, we have $T_{X_{1}} \operatorname{Hilb}^{P(t)}(X) \cong H^{0}\left(\mathcal{N}_{X_{1} / X}, X\right)=H^{0}\left(X, \operatorname{Hom}_{\mathcal{O}_{X_{1}}}\left(\mathcal{I}_{1} / \mathcal{I}_{1}^{2}, \mathcal{O}_{X_{1}}\right)\right)$, where $\mathcal{I}_{1}$ is the ideal sheaf of $X_{1}$. For the proof of the result for flag Hilbert schemes, we refer to [Ser06, Proposition 4.5.3].

The following proposition is proved in, for example, [She12, Proposition 17], and we re-prove it here for the convenience of the reader. 


\section{Homology of Hilbert schemes of Planar CURVES}

Proposition 2.4. Let $\pi: \mathcal{C} \rightarrow B^{\prime}$ be a versal deformation of $C$, a reduced, locally planar curve. Then the total space of the family $\pi^{[n]}: \mathcal{C}^{[n]} \rightarrow B^{\prime}$ is smooth.

Proof. Let $B \subset \mathbb{C}[x, y]$ be a finite-dimensional, smooth family of polynomials containing the local equation for $C$ and all polynomials of degree at most $n$, such that the associated deformation is versal. Consider the family of curves over $B$ given by $\mathcal{C}_{B}:=\left\{\left(f \in B, p \in \mathbb{C}^{2}\right) f(p)=0\right\}$. Denote the fiber over $f$ by $C_{f}$, and let $Z \subset C_{f}$ be a subscheme of length $n$. By, for example, [Ser06, Section 4], there is always an exact sequence

$$
0 \rightarrow H^{0}\left(\mathcal{N}_{Z / C_{f}}, Z\right) \rightarrow T_{Z} C_{B}^{[n]} \rightarrow T_{f} B \rightarrow \operatorname{Ext}_{\mathcal{O}_{C_{f}}}^{1}\left(\mathcal{I}_{Z}, \mathcal{O}_{Z}\right)
$$

For squarefree $f$, there is always some open neighborhood $U$ of $f$ such that $C_{U}^{[n]}$ is reduced of pure dimension $n+\operatorname{dim} B$ (see [MY14, Proposition 3.5]). Since $B$ is smooth and $H^{0}\left(\mathcal{N}_{Z / C_{f}}, Z\right)$ has dimension $n$ (see, for example, [Che98]), it is enough to prove that the last Ext-group vanishes to get the smoothness of the total space $C_{U}^{[n]}$ at $Z$.

Now, from the short exact sequence $0 \rightarrow \mathcal{I}_{Z} \rightarrow \mathcal{O}_{C_{f}} \rightarrow \mathcal{O}_{Z} \rightarrow 0$, taking Hom to $\mathcal{O}_{Z}$, we have

$$
\cdots \rightarrow \operatorname{Ext}_{\mathcal{O}_{C_{f}}}^{1}\left(\mathcal{O}_{C_{f}}, \mathcal{O}_{Z}\right) \rightarrow \operatorname{Ext}_{\mathcal{O}_{C_{f}}}^{1}\left(\mathcal{I}_{Z}, \mathcal{O}_{Z}\right) \rightarrow \operatorname{Ext}_{\mathcal{O}_{C_{f}}}^{2}\left(\mathcal{O}_{Z}, \mathcal{O}_{Z}\right)=0 \rightarrow \cdots
$$

Since $\operatorname{Ext}_{\mathcal{O}_{C_{f}}}^{1}\left(\mathcal{O}_{C_{f}}, \mathcal{O}_{Z}\right) \cong H^{1}\left(\mathcal{O}_{Z}, C_{f}\right)=0$ and the sequence is exact, we must also have $\operatorname{Ext}_{\mathcal{O}_{C_{f}}}^{1}\left(\mathcal{I}_{Z}, \mathcal{O}_{Z}\right)=0$. So the total space is smooth.

Now, if $\overline{\mathcal{C}} \rightarrow \bar{B}$ is the miniversal deformation, by versality there are compatible isomorphisms $\mathcal{C} \cong \overline{\mathcal{C}} \times\left(\mathbb{C}^{t}, 0\right)$ and $B \cong \bar{B} \times\left(\mathbb{C}^{t}, 0\right)$ for some $t$; see, for example, [GLS07]. Hence, we have smoothness for any versal family.

We now consider the relative flag Hilbert scheme of a versal deformation. If $S$ is a smooth complex algebraic surface, its nested Hilbert scheme of points $S^{[n, n+1]}$ is smooth by results of [Che98, Tik97].

Remark 2.5. This nested Hilbert scheme $S^{[n, n+1]}$ and the ordinary Hilbert scheme of $n$ points $S^{[n]}$ are the only flag Hilbert schemes of points on $S$ that are smooth, as shown in [Che98, Tik97].

We also have the following result.

Proposition 2.6. The total space of the relative family $\mathcal{C}^{[n, n+1]} \rightarrow B$ is smooth.

Proof. This is a local question, so we can assume that $C$ is the germ of a plane curve singularity in $\mathbb{C}^{2}$.

From Lemma 2.3, we have that at $(J \subset I) \in \mathcal{C}^{[n, n+1]}$, the tangent space is $\operatorname{ker}(\phi-\psi)$, where

$$
\begin{aligned}
& \phi: \operatorname{Hom}_{\mathbb{C}[x, y]}(I, \mathbb{C}[x, y] / I) \rightarrow \operatorname{Hom}_{\mathbb{C}[x, y]}(J, \mathbb{C}[x, y] / I), \quad \text { and } \\
& \psi: \operatorname{Hom}_{\mathbb{C}[x, y]}(J, \mathbb{C}[x, y] / J) \rightarrow \operatorname{Hom}_{\mathbb{C}[x, y]}(J, \mathbb{C}[x, y] / I)
\end{aligned}
$$

are the induced maps given by restriction and further quotient. Here, $\phi-\psi$ is the difference of the maps from the direct sum. This is precisely the requirement needed for the normal vector fields in question.

Suppose again that $B \subset \mathbb{C}[x, y]$ is a finite-dimensional, smooth family of polynomials containing the local equation for $C$ and all polynomials of degree at most $n+1$, such that the associated deformation is versal. 


\section{O. Kivinen}

Consider the inclusion $\mathcal{C}_{B}^{[n, n+1]} \hookrightarrow B \times\left(\mathbb{C}^{2}\right)^{[n, n+1]}$. We have an exact sequence

$$
0 \rightarrow T_{f, J \subset I} \mathcal{C}_{B}^{[n, n+1]} \rightarrow T_{f} B \times T_{J \subset I}\left(\mathbb{C}^{2}\right)^{[n, n+1]} \rightarrow \mathbb{C}[x, y] / J
$$

where the last map is given by

$$
\left(f+\epsilon g,\left(\begin{array}{l}
\eta_{1} \\
\eta_{2}
\end{array}\right)\right) \mapsto\left(\phi \eta_{1}\right)(f)-g \bmod J
$$

By the assumption on $B$, the last map is surjective; hence, $T_{f, J \subset I} \mathcal{C}_{B}^{[n, n+1]}$ has dimension

$$
\operatorname{dim}\left(\mathbb{C}^{2}\right)^{[n, n+1]}+\operatorname{dim} B-n+1=n+1+\operatorname{dim} B,
$$

as expected, and the total space is smooth.

Again, if $\overline{\mathcal{C}} \rightarrow \bar{B}$ is the miniversal deformation, by versality there are compatible isomorphisms $\mathcal{C} \cong \overline{\mathcal{C}} \times\left(\mathbb{C}^{t}, 0\right)$ and $B \cong \bar{B} \times\left(\mathbb{C}^{t}, 0\right)$ for some $t$; see, for example, [GLS07]. Hence, we have smoothness for any versal family.

Another result we will also need is the description of the components and dimensions of the irreducible components of $C^{[n]}$.

Proposition 2.7. For any locally planar, reduced curve $C=\bigcup_{i=1}^{m} C_{i}$, the irreducible components of $C^{[n]}$ are given by

$$
\overline{\left(C_{1}^{s m}\right)^{\left[r_{1}\right]} \times \cdots \times\left(C_{m}^{s m}\right)^{\left[r_{m}\right]}}, \quad \sum_{i} r_{i}=n .
$$

Here, $C_{i}^{s m}$ denotes the smooth locus of $C_{i}$. In particular, there are $\left(\begin{array}{c}n+m-1 \\ n\end{array}\right)$ irreducible components of $C^{[n]}$, all of dimension $n$.

Proof. That $\left(C^{s m}\right)^{[n]}$ is dense in $C^{[n]}$ can be found, for example, in [MRV17, Fact 2.4]. The schemes $\left(C_{1}^{s m}\right)^{\left[r_{1}\right]} \times \cdots \times\left(C_{m}^{s m}\right)^{\left[r_{m}\right]}$ are disjoint. They are of dimension $n$, smooth and connected, so irreducible, and as $\left(r_{1}, \ldots, r_{m}\right)$ runs over all possibilities, cover $\left(C^{s m}\right)^{[n]}$. Taking closures, we get the result.

\section{Definition of the algebra A}

Let $V=\bigoplus_{i \geqslant 0} H_{*}\left(C^{[n]}, \mathbb{Q}\right)$, where we take the singular homology in the analytic topology. This is mostly for simplicity; a majority of the results work with $\mathbb{Z}$-coefficients. A notable exception is Section 5, where $\mathbb{Q}$-coefficients are essential. From now on, we will be suppressing the coefficients from our notation. The space $V$ is naturally a bigraded $\mathbb{Q}$-vector space, graded by the number of points $n$ and homological degree $d$. We denote the $(n, d)$-graded piece of $V$ by $V_{n, d}$. We define operators on $V$ following ideas of Rennemo [Ren18] (that originally go back to Nakajima and Grojnowski [Nak97, Gro96]).

Definition 3.1. (i) Let $c_{i} \in C_{i}^{s m}$ be fixed smooth points and $\iota_{i}: C^{[n]} \rightarrow C^{[n+1]}$ be the maps $Z \mapsto Z \cup c_{i}$. Let $x_{i}: V \rightarrow V$ be the operators given by $\left(\iota_{i}\right)_{*}$. These are homogeneous of degree $(1,0)$ and depend only on the component the points $c_{i}$ lie in; see Lemma 3.2 below.

(ii) Let $d_{i}: V \rightarrow V$ be the operators given by the Gysin/intersection pullback map $\left(\iota_{i}\right)$ ! These are homogeneous of degree $(-1,-2)$ and well defined since the $\iota_{i}$ are regular embeddings. See Lemma 3.3 below for a proof of this latter fact. 


\section{Homology of Hilbert SCHEMES OF PLANAR CURVES}

Lemma 3.2. The maps $\iota_{c_{i}}$ and $\iota_{c_{i}^{\prime}}$ are homotopic whenever $c_{i}, c_{i}^{\prime} \in C_{i}^{s m}$. In particular, the corresponding pushforwards induce the same operators $x_{i}$ on $V$.

Proof. Take any path $c_{i}(t)$ from $c_{i}$ to $c_{i}^{\prime}$, and consider the homotopy $C^{[n]} \times[0,1] \rightarrow C^{[n+1]}$ given by $(Z, t) \mapsto Z \cup c_{i}(t)$.

LEMma 3.3. The map $\iota_{x}$ is a regular embedding.

Proof. This is a property which is local in the analytic topology [ACG11, Chapter 2, Lemma 2.6]. Suppose that $Z \subset C$ is a subscheme of length $n$ which contains $x$ with multiplicity $k$.

If $U$ is an analytic open set around $x$ such that the only component of $Z$ contained in $\bar{U}$ (the closure in the analytic topology) is $x$, then locally around $Z$, the morphism is isomorphic to

$$
U^{[k]} \times(C \backslash \bar{U})^{[n-k]} \hookrightarrow(U)^{[k+1]} \times(C \backslash \bar{U})^{[n-k]},
$$

where the map is given on factors by adding $x$ and the identity map, respectively. In local coordinates, the first map looks exactly like the inclusion $\mathbb{C}^{[k]} \rightarrow \mathbb{C}^{[k+1]}$ given as follows. If we identify coordinates on $\mathbb{C}^{[k]}$ with symmetric functions $a_{i}$ in the roots of some degree $k$ polynomial, that is, coefficients of a monic polynomial of degree $k$, the map is given by $\sum_{i=0}^{k-1} a_{i} z^{i} \mapsto(z-x) \sum_{i=0}^{k-1} a_{i} z^{i}$. But this last map is linear in the $a_{i}$ and of rank $k$, in particular a regular embedding.

Consider the following diagram:

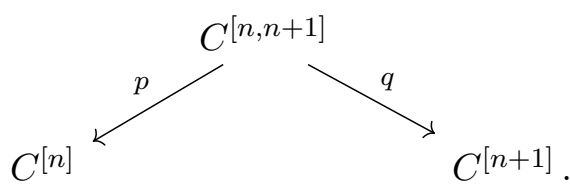

To define the operators $\mu_{+}$and $\mu_{-}$, we want to define correspondences in homology between $C^{[n]}$ and $C^{[n+1]}$. This is done as follows. By Propositions 2.4 and 2.6, we may embed $C$ into a smooth, locally versal family $\pi: \mathcal{C} \rightarrow B$ such that the relative family $\mathcal{C}^{[n]}$ is smooth and $\pi^{-1}(0)=C$. After possibly doing an étale base extension, we may also assume that the family also has sections $s_{i}: B \rightarrow \mathcal{C}$ hitting only the smooth loci of the fibers and such that $s_{i}(0)=c_{i}$.

Now, consider the diagram

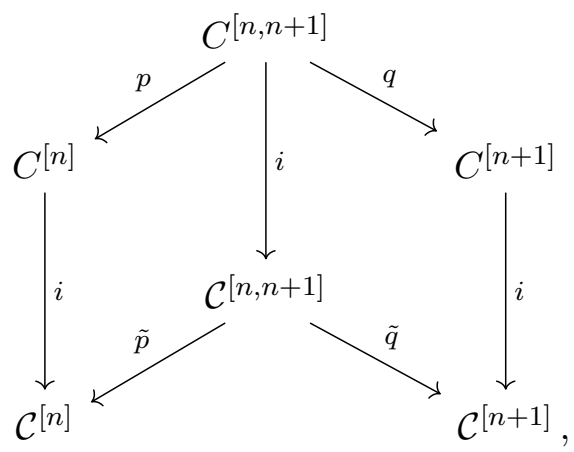

where $i$ is the inclusion of the central fiber. Since $\mathcal{C}^{[n]}$ is smooth, from Property (7) in Section 3.1, we have

$$
H^{*}\left(\mathcal{C}^{[n, n+1]} \rightarrow \mathcal{C}^{[n]}\right) \cong H_{*-2 n-\operatorname{dim} B}^{\mathrm{BM}}\left(\mathcal{C}^{[n, n+1]}\right) .
$$

Denote the fundamental class of $\mathcal{C}^{[n, n+1]}$ under this isomorphism by $[\tilde{p}]$. Then pulling back $[\tilde{p}]$ along $i$ to $H^{*}\left(C^{[n, n+1]} \rightarrow C^{[n]}\right)$ gives us a canonical orientation, using which we define $p^{!}: H_{*}\left(C^{[n]}\right)$ 


\section{O. Kivinen}

$\rightarrow H_{*}\left(C^{[n, n+1]}\right)$ as $p^{!}(\alpha)=\alpha \cdot i^{*}([\tilde{p}])$. The definition of $q^{!}$is analogous, where we replace $C^{[n]}$ with $C^{[n+1]}$.

We are finally ready to define the operators $\mu_{+}$and $\mu_{-}$.

Definition 3.4. Let $\mu_{ \pm}: V \rightarrow V$ be the Nakajima correspondences $\mu_{+}=q_{*} p^{!}$and $\mu_{-}=p_{*} q^{!}$. These are operators of respective bidegrees $(1,2)$ and $(-1,0)$.

Remark 3.5. The $n$-degree in the above maps is easy to see from the definition. The homological degrees follow from the definition of the Gysin maps using $i^{*}[\tilde{p}]$, which sits in homological degree $2 n+2$, and the fact that degrees are additive under the bivariant product.

We are now ready to define the algebra(s) $A$.

Theorem 3.6. The operators from Definitions 3.1 and 3.4 satisfy the following commutation relations: $\left[d_{i}, \mu_{+}\right]=\left[\mu_{-}, x_{i}\right]=1$, and the rest are trivial.

Remark 3.7. For $m=1$, we recover [Ren18, Theorem 1.2].

Definition 3.8. Fix $m \geqslant 1$. Let $A_{m}$ be the $\mathbb{Q}$-algebra generated by the symbols

$$
x_{1}, \ldots, x_{m}, d_{1}, \ldots, d_{m}, \mu_{+}, \mu_{-}
$$

with the relations

$$
\left[d_{i}, \mu_{+}\right]=\left[\mu_{-}, x_{i}\right]=1, \quad\left[x_{i}, x_{j}\right]=\left[x_{i}, d_{i}\right]=\left[x_{i}, \mu_{+}\right]=\left[d_{i}, \mu_{-}\right]=0 .
$$

Remark 3.9. We can realize $A_{m}$ inside Weyl $\left(\mathbb{A}_{\mathbb{Q}}^{2 m}\right)$ as follows: Let $\mathbb{A}^{2 m}$ have coordinates $x_{1}, \ldots$, $x_{m}, y_{1}, \ldots, y_{m}$ and $d_{i}=\partial_{y_{i}}, k=\sum_{i=1}^{m} \partial_{x_{i}}, j=\sum_{i=1}^{m} y_{i}$. Then from the commutation relations, we immediately have that $A_{m}$ is isomorphic to the subalgebra $\left\langle x_{i}, \partial_{y_{i}}, \sum_{i=1}^{m} \partial_{x_{i}}, \sum_{i=1}^{m} x_{i}\right\rangle \subset$ $\operatorname{Weyl}\left(\mathbb{A}_{\mathbb{Q}}^{2 m}\right)$.

Remark 3.10. Although $A_{m}$ depends on $m$, we will be suppressing the subscript from the notation from here on. It should be evident from the context which $m$ we are considering.

Let us give an outline of the proof of Theorem 3.6. We first prove the trivial commutation relations in Subsections 4.1 and 4.2. In Subsection 4.3, we then prove that $\left[d_{i}, \mu_{+}\right]=1$, with the aid of the bivariant homology formalism, and then in a similar vein that $\left[\mu_{-}, x_{i}\right]=1$.

\subsection{Bivariant Borel-Moore homology}

We now describe the bivariant Borel-Moore homology formalism from [FM81]. Suppose that we are in a category of "nice" spaces, for example, those that can be embedded in some $\mathbb{R}^{n}$. We will not define bivariant homology here, but for us, the most essential facts about it are the following ones:

(1) The theory associates with maps $X \stackrel{f}{\rightarrow} Y$ a graded abelian group $H^{*}(X \stackrel{f}{\rightarrow} Y)$. We will be working over $\mathbb{Q}$ throughout and with bivariant homology.

(2) Given maps $X \stackrel{f}{\rightarrow} Y \stackrel{g}{\rightarrow} Z$, there is a product homomorphism

$$
H^{i}(X \stackrel{f}{\rightarrow} Y) \otimes H^{j}(Y \stackrel{g}{\rightarrow} Z) \rightarrow H^{i+j}(X \stackrel{g \circ f}{\rightarrow} Z) .
$$

For $\alpha \in H^{i}(X \stackrel{f}{\rightarrow} Y)$ and $\beta \in H^{j}(Y \stackrel{g}{\rightarrow} Z)$, we thus get a product $\alpha \cdot \beta \in H^{i+j}(X \stackrel{g \circ f}{\longrightarrow} Z)$.

(3) For any proper map $X \stackrel{f}{\rightarrow} Y$ and any map $Y \stackrel{g}{\rightarrow} Z$, there is a pushforward homomorphism $f_{*}: H^{*}(X \stackrel{g \circ f}{\longrightarrow} Z) \rightarrow H^{*}(Y \stackrel{g}{\rightarrow} Z)$. 
(4) For any cartesian square

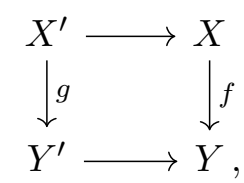

there is a pullback homomorphism $H^{*}(X \stackrel{f}{\rightarrow} Y) \rightarrow H^{*}\left(X^{\prime} \stackrel{g}{\rightarrow} Y^{\prime}\right)$. (Recall that a cartesian square is a square where $X^{\prime} \cong X \times_{Y} Y^{\prime}$.)

(5) The product and pullback commute: given a tower of cartesian squares

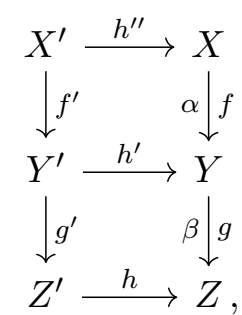

we have $h^{*}(\alpha \cdot \beta)=h^{*}(\alpha) \cdot h^{*}(\beta)$ in $H^{*}\left(X^{\prime} \stackrel{g^{\prime} \circ f^{\prime}}{\longrightarrow} Z^{\prime}\right)$.

(6) The product and pushforward commute: given

$$
X \stackrel{f}{\rightarrow} Y \stackrel{g}{\rightarrow} Z \stackrel{h}{\rightarrow} W
$$

with $\alpha \in H^{*}(X \stackrel{g \circ f}{\longrightarrow} Z)$ and $\beta \in H^{*}(Z \stackrel{h}{\rightarrow} W)$, we have $f_{*}(\alpha \cdot \beta)=f_{*}(\alpha) \cdot \beta$ in $H^{*}(Y \stackrel{h \circ g}{\longrightarrow} W)$.

(7) For any space $X$, the groups $H^{i}(X \rightarrow \mathrm{pt})$ and $H^{i}(X \stackrel{\text { id }}{\rightarrow} X)$ are by construction canonically identified with $H_{-i}^{\mathrm{BM}}(X)$ and $H^{i}(X)$, respectively. These are called the associated covariant and contravariant theories, respectively. Note that the three bivariant operations recover the usual homological operations of cup and cap product, proper pushforwards in homology and arbitrary pullbacks in cohomology.

(8) If $Y$ is a nonsingular variety and $f: X \rightarrow Y$ is any morphism, the induced homomorphism

$$
H^{*}(X \stackrel{f}{\rightarrow} Y) \rightarrow H^{*-2 \operatorname{dim} Y}(X \rightarrow \mathrm{pt})=H_{2 \operatorname{dim} Y-*}^{\mathrm{BM}}(X)
$$

given by taking the product with $[Y] \in H^{-2 \operatorname{dim} Y}(Y \rightarrow \mathrm{pt})$ is an isomorphism. Again, the last equality is given by the associated covariant theory. In such a situation, we will frequently identify $H^{*}(X \rightarrow Y)$ with $H_{2 \operatorname{dim} Y-*}^{\mathrm{BM}}(X)$. In particular, if $X$ has a fundamental class $[X] \in H_{2 \operatorname{dim} X}^{\mathrm{BM}}(X)$, this induces a class $[X] \in H^{2(\operatorname{dim} Y-\operatorname{dim} X)}(X \rightarrow Y)$.

(9) Any class $\alpha \in H^{i}(X \stackrel{f}{\rightarrow} Y)$ defines a Gysin pullback map $f^{!}: H_{*}^{\mathrm{BM}}(Y) \rightarrow H_{*-i}^{\mathrm{BM}}(X)$ by

$$
f^{!}(\beta):=\alpha \cdot \beta, \quad \forall \beta \in H_{*}^{\mathrm{BM}}(Y) .
$$

\section{Proof of the commutation relations}

\subsection{Proof of the trivial commutation relations for $x_{i}$ and $d_{i}$}

We now show that $\left[x_{i}, x_{j}\right]=0$ for all $i, j$. This is fairly easy; under either composition $\iota_{i} \circ \iota_{j}$ or $\iota_{j} \circ \iota_{i}$, we map $Z \mapsto Z \cup x_{i} \cup x_{j}$ and as $\left(\iota_{i} \circ \iota_{j}\right)_{*}=x_{i} x_{j}$, we get $x_{i} x_{j}=x_{j} x_{i}$.

The next step is to describe the Gysin maps and their commutation relations. Denote these as before by $d_{i}=\left(\iota_{x_{i}}\right)^{!}: H_{*}\left(C^{[n]}\right) \rightarrow H_{*-2}\left(C^{[n-1]}\right)$. 


\section{O. Kivinen}

Proposition 4.1. We have $\left[d_{i}, d_{j}\right]=0$ and $\left[d_{i}, x_{j}\right]=0$.

Proof. Let $\alpha \in H_{*}\left(C^{[n]}\right)$. As we saw before, $\iota_{x_{i}} \circ \iota_{x_{j}}=\iota_{x_{j}} \circ \iota_{x_{i}}$. By the functoriality of the Gysin maps, $\left[d_{i}, d_{j}\right]=0$. Now, choosing a representative for $\alpha$, we see that

$$
d_{i} x_{j}(\alpha)=\left(\iota_{j}\right)^{!}\left[\iota_{i}(\alpha)\right]=\left[Z \in \alpha \mid c_{i}, c_{j} \subset Z\right] .
$$

However, we also have

$$
x_{j} d_{i}(\alpha)=x_{j}\left[Z^{\prime} \in \alpha \mid c_{i} \subset Z^{\prime}\right]=\left[Z \in \alpha \mid c_{i}, c_{j} \subset Z\right] .
$$

When the points are equal in the above, that is to say $i=j$, we pick a linearly equivalent point $c_{i}^{\prime}$ near $c_{i}$. Since the inclusion maps $\iota_{c_{i}}$ and $\iota_{c_{i}^{\prime}}$ are homotopic in this case by Lemma 3.2, we still have $\left[d_{i}, x_{j}\right]=0$.

\subsection{Proof of the trivial commutation relations for Nakajima operators}

As we saw before, the definition of the Nakajima operators requires making sense of the Gysin morphisms $p^{\text {! }}$ and $q^{\text {! }}$, which is done using the bivariant homology formalism. Recall that we are working with a fixed family $\mathcal{C} \rightarrow B$ as in Section 3, guaranteeing the smoothness of $\mathcal{C}^{[n]}$ and $\mathcal{C}^{[n, n+1]}$. In this section and later on, all commutative diagrams should be thought of as commutative diagrams of topological spaces (corresponding to the analytic spaces of the varieties under consideration) and living over $B$, so that we may restrict to the central fiber and obtain similar squares with the calligraphic $\mathcal{C}$ replaced with the regular $C$, that is, our curve of interest. We will denote these restrictions in homology computations by the subscript 0 .

Proposition 4.2. We have $\left[x_{i}, \mu_{+}\right]=0$ and $\left[d_{i}, \mu_{-}\right]=0$.

Proof. Consider the following commutative diagram:

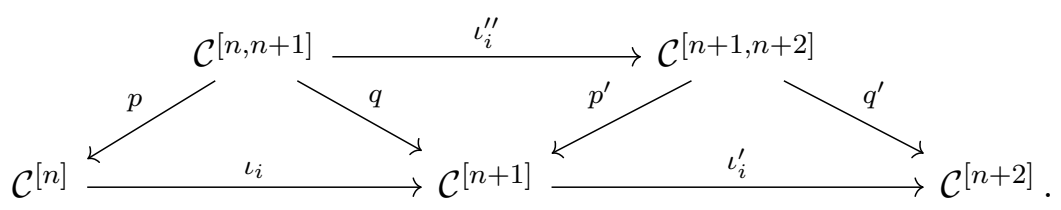

Here, $\iota_{i}$ and $\iota_{i}^{\prime}$ are defined as adding points at the sections $s_{i}$ to $\mathcal{C}^{[n]}$ and $\mathcal{C}^{[n+1]}$, respectively, and $\iota_{i}^{\prime \prime}$ is adding points at the section $s_{i}$ as follows: $\left(Z_{1} \subset Z_{2}\right) \mapsto\left(Z_{1} \cup s_{i} \subset Z_{2} \cup s_{i}\right)$.

We have, by definition, $x_{i} \mu_{+}=\left(\left(\iota_{s_{i}}^{\prime}\right)_{*} q_{*} p^{!}\right)_{0}$, where the subscript 0 denotes restriction to the central fiber.

In diagram (4.1), the square formed by the maps $\iota_{i}^{\prime}, q, q^{\prime}$ and $\iota_{i}^{\prime \prime}$ is commutative, so on homology, we have $\left(\iota_{i}^{\prime}\right)_{*} q_{*} p^{!}=q_{*}^{\prime}\left(\iota_{i}^{\prime \prime}\right)_{*} p^{!}$. Similarly, the square formed by the maps $\iota_{i}, p^{\prime}, p$, $\iota_{i}^{\prime \prime}$ is commutative. Because of the fact that the pushforward and the Gysin maps in bivariant homology also commute in this case, as explained in Section 3.1 (Property $(7)$ ), we get $q_{*}^{\prime}\left(\iota_{i}^{\prime \prime}\right)_{*} p^{!}=$ $q_{*}^{\prime}\left(p^{\prime}\right)^{!}\left(\iota_{i}\right)_{*}$. Restricting to $C$, we have $\left(q_{*}^{\prime}\left(p^{\prime}\right)^{!}\left(\iota_{i}\right)_{*}\right)_{0}=\mu_{+} x_{i}$. Similarly, for the other commutation relation we have $\mu_{-} d_{i}=\left(p_{*} q^{!}\left(\iota_{i}^{\prime}\right)^{!}\right)_{0}=\left(p_{*}\left(\iota_{i}^{\prime \prime}\right)^{!}\left(q^{\prime}\right)^{!}\right)_{0}=\left(\left(\iota_{i}\right)^{!} p_{*}^{\prime}\left(q^{\prime}\right)^{!}\right)_{0}=d_{i} \mu_{-}$.

Let us explain the restriction to the central fiber once and for all. For example, in the last computation, if $\alpha \in H_{*}\left(C^{[n]}\right)$, we have $\mu_{-} d_{i} \alpha=i^{*}[\tilde{p}] \cdot \iota_{i}^{\vdots} \alpha=i^{*}[\tilde{p}] \cdot i^{*}\left[\tilde{d}_{i}\right] \cdot \alpha$, where $i^{*}\left[\tilde{d}_{i}\right]=\left[d_{i}\right]$ is the fundamental class corresponding to the Gysin map $\iota_{i}^{!}$and $\left[\tilde{d}_{i}\right]$ is the corresponding class in the family. Since the product and pullback commute in the bivariant theory, $i^{*}[\tilde{p}] \cdot i^{*}\left[\tilde{d}_{i}\right] \cdot \alpha=$ $i^{*}\left([\tilde{p}] \cdot\left[\tilde{d}_{i}\right]\right) \cdot \alpha$. Similarly, $d_{i} \mu_{-} \alpha=\left[d_{i}\right] \cdot i^{*}[\tilde{p}] \cdot \alpha=i^{*}\left(\left[\tilde{d}_{i}\right] \cdot[\tilde{p}]\right) \cdot \alpha$. So composing our operators in the family and then deducing the result for $C$ is justified. 


\section{Homology of Hilbert SCHEMES OF PLANAR CURVES}

4.3 Proof that $\left[d_{i}, \mu_{+}\right]=\left[\mu_{-}, x_{i}\right]=1$

To compute the desired commutation relation, we compare the composition of the operators $d_{i}$ and $\mu_{+}$on $V$ in either order. By abuse of notation we will first consider $d_{i}$ and $\mu_{+}$as operators acting on the space $\mathcal{V}=\bigoplus_{n \geqslant 0} H_{*}^{\mathrm{BM}}\left(\mathcal{C}^{[n]}\right)$ and then use the properties of the bivariant theory, more precisely, the ability to pull back in cartesian squares (Property (5) in Section 3.1), to restrict to the special fiber and get an action on $V$.

Consider the diagrams

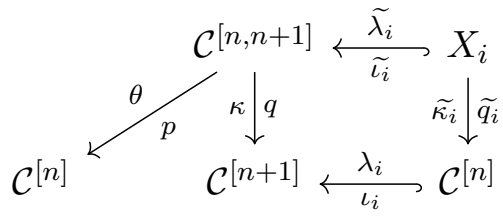

and

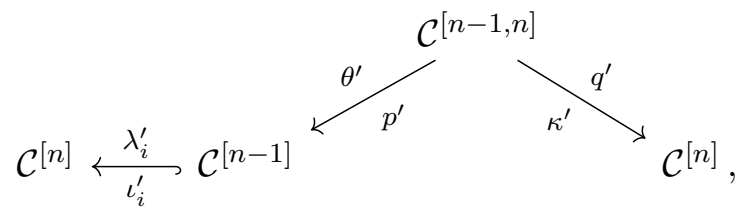

where the two labels on the arrows denote the corresponding map $f: Y \rightarrow Z$ and a bivariant class $\alpha \in H^{*}(X \stackrel{f}{\rightarrow} Y)$.

In the first diagram, $X_{i}=\mathcal{C}^{[n, n+1]} \times_{\mathcal{C}^{[n+1]}} \mathcal{C}^{[n]}$ is the fiber product, and the square containing $X_{i}$ is cartesian. The morphisms $\iota_{i}$ and $\iota_{i}^{\prime}$ correspond to adding a point at the sections $s_{i}: B \rightarrow \mathcal{C}$. The bivariant classes $\theta, \lambda_{i}, \kappa$ and their primed versions are the ones defined by fundamental classes, using the fact that the targets are smooth. The classes $\widetilde{\lambda}_{i}$ and $\widetilde{\kappa_{i}}$ are the cartesian pullbacks of $\lambda_{i}$ and $\kappa$, respectively.

Let $\alpha \in H_{*}\left(\mathcal{C}^{[n]}\right)$. We first compute

$$
\begin{aligned}
& d_{i} \mu_{+}(\alpha)=\lambda_{i}^{\prime} \cdot q_{*}(\theta \cdot \alpha)=\tilde{q}_{*}\left(\tilde{\lambda}_{i} \cdot \theta \cdot \alpha\right), \\
& \mu_{+} d_{i}(\alpha)=q_{*}^{\prime}\left(\theta^{\prime} \cdot \lambda_{i}^{\prime} \cdot \alpha\right) .
\end{aligned}
$$

Let us elaborate a little bit on the first computation. Here, $\theta$ is the fundamental class, and the isomorphism $H_{*-n-1}^{\mathrm{BM}}\left(\mathcal{C}^{[n, n+1]}\right) \cong H^{*}\left(\mathcal{C}^{[n, n+1]} \rightarrow \mathcal{C}^{[n]}\right)$ of the Borel-Moore homology group with the bivariant one is given by the product with the fundamental class $\theta$. On the other hand, the Gysin pullback $\iota_{i}^{!}$is, by definition, equal to the product with $\lambda_{i}$ in the bivariant theory. In the first equation of (4.4), we then use that the diagram (4.2) is cartesian.

Let $f_{i}: \mathcal{C}^{[n-1, n]} \rightarrow X_{i}$ be given by $\left(Z_{1} \subset Z_{2}\right) \mapsto\left(Z_{1} \cup s_{i} \subset Z_{2} \cup s_{i}, Z_{1} \cup s_{i}\right)$ and $g_{i}: \mathcal{C}^{[n]} \rightarrow X_{i}$ be given by $Z \mapsto\left(Z \subset Z \cup s_{i}, Z\right)$.

LEMma 4.3. For all $i$, we have $\left[X_{i}\right]=\left(f_{i}\right)_{*}\left(\left[\mathcal{C}^{[n-1, n]}\right]\right)+\left(g_{i}\right)_{*}\left(\left[\mathcal{C}^{[n]}\right]\right)$.

Proof. By Propositions 2.4 and 2.6, the total spaces of the relative families $\mathcal{C}^{[n]} \rightarrow B$ and $\mathcal{C}^{[n, n+1]} \rightarrow B$ are smooth.

Consider the fiber product $X_{i}$. The images of $f_{i}$ and $g_{i}$ cover all of $X_{i}$. On the level of points (of the fibers) this is easy to see: We are looking at pairs consisting of a flag of subschemes of lengths $n$ and $n+1$ and a subscheme of length $n$ that project to the same length $n+1$ subscheme in the cartesian square (4.2). Since the points in the image contain $s_{i}$, the above pairs come either from adding $s_{i}$ to both parts of the flag as well as taking the second factor to be $Z_{1} \cup s_{i}$, or from creating a new flag by adding $s_{i}$ to $Z$ and taking $Z$ to be the second factor. 


\section{O. Kivinen}

By [Ren18, Lemma 3.4], the intersection of the images of $f_{i}$ and $g_{i}$ is of codimension one in $X_{i}$. Consider a point $\left(Z \subset Z \cup s_{i}, Z\right) \in \operatorname{Im}\left(f_{i}\right) \cap \operatorname{Im}\left(g_{i}\right)$, which can also be written as $\left(Z^{\prime} \cup s_{i} \subset Z^{\prime} \cup\right.$ $\left.s_{i} \cup s_{i}, Z^{\prime} \cup s_{i}\right)$. We can then remove the smooth point $s_{i}$ from both of the factors unambiguously. So the intersection is isomorphic to $\mathcal{C}^{[n-1]}$. On the complement of the intersection, the maps $f_{i}$ and $g_{i}$ are scheme-theoretic isomorphisms because we can unambiguously remove the point $s_{i}$ from $\left(Z \subset Z \cup s_{i}, Z\right)$ or $\left(Z_{1} \cup s_{i} \subset Z_{2} \cup s_{i}, Z_{1} \cup s_{i}\right)$. Hence, the images of $f_{i}$ and $g_{i}$ yield a partition of $X_{i}$ to irreducible components. In particular, the fundamental class $\left[X_{i}\right]$ is the sum of the fundamental classes of the images, which are by definition the pushforwards in question.

Corollary 4.4. We have $\left[X_{i}\right]=\left(f_{i}\right)_{*}\left(\theta^{\prime} \cdot \lambda_{i}^{\prime}\right)+\left(g_{i}\right)_{*}\left[\mathcal{C}^{[n]}\right]$.

Proof. By Lemma 4.3 , we have $\left[X_{i}\right]=\left(f_{i}\right)_{*}\left(\left[\mathcal{C}^{[n-1, n]}\right]\right)+\left(g_{i}\right)_{*}\left(\left[\mathcal{C}^{[n]}\right]\right)$. Rewrite $\left[\mathcal{C}^{[n-1, n]}\right]$ as follows. First of all, note that

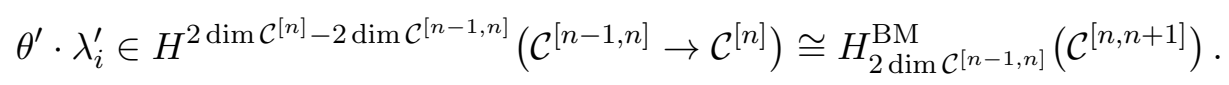

The last isomorphism is given by Property (8) in Section 3.1, that is, taking the product with $\left[\mathcal{C}^{[n]}\right] \in H^{*}\left(\mathcal{C}^{[n]} \rightarrow \mathrm{pt}\right)$. On the other hand, we know that $\theta^{\prime} \cdot \lambda_{i}^{\prime} \cdot\left[\mathcal{C}^{[n]}\right]$ has to be $\left[\mathcal{C}^{[n-1, n]}\right]$ by the same isomorphism. Plugging this into the result of Lemma 4.3 gives $\left[X_{i}\right]=\left(f_{i}\right)_{*}\left(\theta^{\prime} \cdot \lambda_{i}^{\prime}\right)+$ $\left(g_{i}\right)_{*}\left(\left[\mathcal{C}^{[n]}\right]\right)$.

Using Corollary 4.4, we have

$$
\begin{aligned}
d_{i} \mu_{+}(\alpha) & =\left(\tilde{q}_{i}\right)_{*}\left(\tilde{\lambda}_{i} \cdot \theta \cdot \alpha\right)=\left(\tilde{q}_{i}\right)_{*}\left(\left[X_{i}\right] \cdot \alpha\right) \\
& =\left(\tilde{q}_{i}\right)_{*}\left(\left(f_{i}\right)_{*}\left(\theta^{\prime} \cdot \lambda_{i}^{\prime}\right) \cdot \alpha\right)+\left(\tilde{q}_{i}\right)_{*}\left(\left(g_{i}\right)_{*}\left[\mathcal{C}^{[n]}\right] \cdot \alpha\right) .
\end{aligned}
$$

The last equality follows by the linearity of the pushforward. From Property (6) in Section 3.1, we have that the pusforward is also functorial and commutes with products. Hence, we have

$$
\left(\tilde{q}_{i}\right)_{*}\left(\left(f_{i}\right)_{*}\left(\theta^{\prime} \cdot \lambda_{i}^{\prime}\right) \cdot \alpha\right)+\left(\tilde{q}_{i}\right)_{*}\left(\left(g_{i}\right)_{*}\left[\mathcal{C}^{[n]}\right] \cdot \alpha\right)=\left(\tilde{q}_{i} \circ\left(f_{i}\right)\right)_{*}\left(\theta^{\prime} \cdot \lambda_{i}^{\prime} \cdot \alpha\right)+\left(\tilde{q}_{i} \circ\left(g_{i}\right)\right)_{*}(\alpha) .
$$

Since $\tilde{q}_{i} \circ f_{i}=q^{\prime}$, we get $\left(\tilde{q}_{i} \circ\left(f_{i}\right)\right)_{*}\left(\theta^{\prime} \cdot \lambda_{i}^{\prime} \cdot \alpha\right)=q_{*}^{\prime}\left(\theta^{\prime} \cdot \lambda_{i}^{\prime} \cdot \alpha\right)$. Finally, since $\tilde{q}_{i} \circ\left(g_{i}\right)=\mathrm{id}$, we have $\left(\tilde{q}_{i} \circ\left(g_{i}\right)\right)_{*}(\alpha)=\operatorname{id}_{*}(\alpha)$. Substituting these into (4.7), we get

$$
\left(\tilde{q}_{i} \circ\left(f_{i}\right)\right)_{*}\left(\theta^{\prime} \cdot \lambda_{i}^{\prime} \cdot \alpha\right)+\left(\tilde{q}_{i} \circ\left(g_{i}\right)\right)_{*}(\alpha)=q_{*}^{\prime}\left(\theta^{\prime} \cdot \lambda_{i}^{\prime} \cdot \alpha\right)+\operatorname{id}_{*}(\alpha)=\mu_{+} d_{i}(\alpha)+\alpha .
$$

Now, suppose that $\alpha_{0}$ is a class in $H_{*}\left(C^{[n]}\right)$. Then by the fact that pushforward, pullback, and the product in the bivariant theory commute, $\left(\left(\tilde{q}_{i}\right)_{0}\right)_{*}\left(\left(\tilde{\lambda}_{i}\right)_{0} \cdot \theta_{0} \cdot \alpha_{0}\right)=\left(q_{0}^{\prime}\right)_{*}\left(\left(\theta^{\prime}\right)_{0} \cdot\left(\lambda_{i}^{\prime}\right)_{0}\right.$. $\left.\alpha_{0}\right)+\mathrm{id}_{*}\left(\alpha_{0}\right)$ and $d_{i} \mu_{+}=\mu_{+} d_{i}+\mathrm{id}: V \rightarrow V$, as desired.

The case of $\left[\mu_{-}, x_{i}\right]$ is very similar; here, we have

$$
\begin{gathered}
\mu_{-} x_{i}(\alpha)=(p)_{*}\left(\kappa \cdot\left(\iota_{i}\right)_{*}(\alpha)\right)=\left(p \circ \widetilde{\iota}_{i}\right)_{*}\left(\widetilde{\kappa}_{i} \cdot \alpha\right), \\
x_{i} \mu_{-}(\alpha)=\left(\iota_{i}^{\prime} \circ p^{\prime}\right)_{*}\left(\kappa^{\prime} \cdot \alpha\right) .
\end{gathered}
$$

Under the identification of $H^{*}\left(X \stackrel{\widetilde{q}}{\rightarrow} \mathcal{C}^{[n]}\right)$ with $H_{*+2 \operatorname{dim} \mathcal{C}^{[n]}}^{\mathrm{BM}}(X)$, we have $\widetilde{\kappa}_{i}=\left[X_{i}\right]$. This follows from

$$
\widetilde{\kappa}_{i} \cdot\left[\mathcal{C}^{[n]}\right]=\widetilde{\kappa}_{i} \cdot \lambda_{i} \cdot\left[\mathcal{C}^{[n+1]}\right]=\widetilde{\lambda}_{i} \cdot \kappa\left[\mathcal{C}^{[n+1]}\right]=\widetilde{\lambda}_{i} \cdot\left[\mathcal{C}^{[n, n+1]}\right]=\left[X_{i}\right]
$$

where the last equality is the fact that $X_{i}$ is a Cartier divisor in $\mathcal{C}^{[n, n+1]}$. Using Lemma 4.3, we get

$$
\widetilde{\kappa}_{i}=\left[X_{i}\right]=\left(f_{i}\right)_{*}\left[\mathcal{C}^{[n-1, n]}\right]+\left(g_{i}\right)_{*}\left[\mathcal{C}^{[n]}\right]=\left(f_{i}\right)_{*}\left(\kappa^{\prime}\right)+\left(g_{i}\right)_{*}\left[\mathcal{C}^{[n]}\right]
$$




\section{Homology of Hilbert SCHEMES OF PLANAR CURVES}

A computation similar to (4.6) now shows that we have $\mu_{-} x_{i}(\alpha)=x_{i} \mu_{-}(\alpha)+\alpha$, as needed, and the restriction to the special fiber works exactly the same way. This finishes the proof of Theorem 3.6 and thus of Theorem 1.1.

\section{Example: The node}

In this section, we describe the representation $V$ for the curve $\{x y=0\} \subseteq \mathbb{P}_{\mathbb{C}}^{2}$, which is the first nontrivial curve singularity with two components.

\subsection{Geometric description of $C^{[n]}$}

One first thing we may ask is how the components in Proposition 2.7 look. Ran [Ran05b] describes the geometry of the Hilbert scheme of points on (germs of) nodal curves very thoroughly. For $n=0,1$, we get a point and $C$ itself, whereas $C^{[2]}$ is a chain of three rational surfaces that intersect their neighbors transversely along projective lines. More generally, $C^{[n]}$ is a chain of $n+1$ irreducible components of dimension $n$, consecutive members of which meet along codimension one subvarieties.

Lemma 5.1. Denote by $M_{n, k}$ the irreducible component of $C^{[n]}$, where generically we have $k$ points on the component $y=0$ of $C$ and $n-k$ points on the component $x=0$. Then

$$
M_{n, k} \cong \mathrm{Bl}_{\mathbb{P}^{k-1} \times \mathbb{P}^{n-k-1}}\left(\mathbb{P}^{k} \times \mathbb{P}^{n-k}\right) .
$$

Proof. First of all, $\mathbb{P}^{k} \times \mathbb{P}^{n-k}$ has natural coordinates given by the coefficients of polynomials $(a(x), b(y))$ of degrees $k$ and $n-k$. It is also natural to identify the roots of these polynomials with the corresponding subschemes in $C_{1}, C_{2} \cong \mathbb{P}^{1}$. From $(a(x), b(x))$, we construct an ideal in the homogeneous coordinate ring of $C$ by taking the product

$$
I=(y, a(x))(x, b(x))=(x y, x a(x), y b(y), a(x) b(y)) .
$$

This determines a length $n$ subscheme, so a point in $M_{n, k}$. Note that this map is invertible outside the locus where we have at least one point from each axis at the origin.

We can further write $a(x) b(y)=a_{0} b_{0}+a_{0} b^{\prime}(y)+b_{0} a^{\prime}(x) \bmod (x y)$, where $a(x)=a_{0}+a^{\prime}(x)$, $b(y)=b_{0}+b^{\prime}(y)$ and $a^{\prime}(x), b^{\prime}(x)$ have no constant term. Now, consider the limit of $I=I_{1}$ as the products of the coordinates of the roots of $a(x)$ and $b(y)$ separately go to zero linearly; that is, let $t \rightarrow 0$ in $a_{0}=A t, b_{0}=B t$ and in the corresponding family of ideals $I_{t}$. Since this is a flat family, the limiting ideal $I_{0}=\lim _{t \rightarrow 0} I_{t}$ has the same colength and support on the locus where at least one point from each axis is at the origin. In particular, $\left(a_{0} b_{0}+a_{0} b^{\prime}(y)+b_{0} a^{\prime}(x)\right) / t \rightarrow A b^{\prime}(y)+B a^{\prime}(x)$ as $t \rightarrow 0$, and

$$
\lim _{t \rightarrow 0} I_{t}=\left(x y, x a^{\prime}(x), y b^{\prime}(y), A b^{\prime}(y)+B a^{\prime}(x)\right) .
$$

Since all ideals in the locus of $M_{n, k}$ with at least one point from each axis at the origin can be written in this form and $(A: B) \in \mathbb{P}^{1}$ determines the limiting ideal completely, we can identify $(A: B)$ with the normal coordinates $\left(a_{0}: b_{0}\right)$ and the natural map

$$
\pi: M_{n, k}=\overline{\left(C_{1}^{s m}\right)^{[k]} \times\left(C_{2}^{s m}\right)^{[n-k]}} \rightarrow \mathbb{P}^{k} \times \mathbb{P}^{n-k}
$$

is the blowup along the locus where both $a(x)$ and $b(y)$ have zero as a root.

See also [Ran05a] for a similar blowup description.

The intersections of the components can also be seen in this description. 


\section{O. Kivinen}

LEMmA 5.2. We have $E_{k, k+1}^{n}=M_{n, k} \cap M_{n, k+1} \cong \mathbb{P}^{n-k-1} \times \mathbb{P}^{k}$, and all the other intersections are trivial.

Proof. We continue in the notation of the proof of Lemma 5.1. Denote the locus of $M_{n, k}$ where at least one point from either axis is at the origin by $L_{n, k}$. Then, suppose that we are outside $L_{n, k} \cup L_{n, \tilde{k}}$ inside $M_{n, k} \cap M_{n, \tilde{k}}$. Then only one point is at the origin, and this locus is naturally identified with the complement of the corresponding locus in $\mathbb{P}^{n-k-1} \times \mathbb{P}^{k}$ if $k+1=\tilde{k}$ and is empty otherwise. We are thus left to studying the loci $L_{n, k}$.

Consider again points $\left.I=\left(x y, x a^{\prime}(x), y b^{\prime}(y)\right), A b^{\prime}(y)+B a^{\prime}(x)\right)$ in $L_{n, k}$ and points $\tilde{I}=$ $\left.\left(x y, x \tilde{a}^{\prime}(x), y \tilde{b}^{\prime}(y)\right), \tilde{A} \tilde{b}^{\prime}(y)+\tilde{B} \tilde{a}^{\prime}(x)\right)$ in $L_{n, \tilde{k}}$. First, restrict $I$ to the $x$-axis; that is, let $y=0$. Then $\left.I\right|_{y=0}=\left(x a^{\prime}(x), B a^{\prime}(y)\right)$. If $B=0$, this has colength $k+1$ since $a^{\prime}(x)$ is of degree $k$. If $B \neq 0$, the colength is $k$. Similarly, we get the colengths of $\left.\tilde{I}\right|_{y=0}$ to be $\tilde{k}$ or $\tilde{k}+1$ depending on whether $\tilde{B}$ is nonzero or not. Without loss of generality, we can assume $\tilde{k}>k$. In this case, the only possibility for $I$ and $\tilde{I}$ to be in the intersection $M_{n, k} \cap M_{n, k+1}$ is to have $k+1=\tilde{k}, B=0$ and $\tilde{B} \neq 0$. A similar analysis for the $y$-axis shows that we must have $\tilde{A}=0$ and $A \neq 0$. So in particular, the intersections $E_{k, \tilde{k}}^{n}$ are isomorphic to $\mathbb{P}^{n-k-1} \times \mathbb{P}^{k}$ if $k+1=\tilde{k}$ and empty otherwise.

Now, one may compute $V$. There is a natural stratification of a blowup to the exceptional divisor and its complement. These both come with affine pavings, so a particularly easy way to compute the cohomologies of $C^{[n]}$, or at least the Betti numbers, is to count these cells.

Proposition 5.3. The bigraded Poincaré series for the space $V=\bigoplus_{n \geqslant 0} H_{*}\left(C^{[n]}\right)$ is given by

$$
P_{V}(q, t)=\frac{q^{2} t^{2}-q+1}{(1-q)^{2}\left(1-q t^{2}\right)^{2}} .
$$

The grading corresponding to $t$ is the homological degree, whereas $q$ keeps track of the grading given by the number of points.

Proof. It is easily confirmed that the Poincaré polynomials of the components are given by

$$
P_{M_{n, k}}(t)=t^{2}\left(\sum_{i=0}^{k-1} t^{2 i}\right)\left(\sum_{i=0}^{n-k-1} t^{2 i}\right)+\left(\sum_{i=0}^{n-k} t^{2 i}\right)\left(\sum_{i=0}^{k} t^{2 i}\right) .
$$

Similarly, the Poincaré polynomials of the intersections are given by

$$
Q_{E_{k, k+1}^{n}}(t)=\left(\sum_{i=0}^{k} t^{2 i}\right)\left(\sum_{i=0}^{n-k-1} t^{2 i}\right), \quad k \leqslant n-1,
$$

and by the Mayer-Vietoris sequence, $\sum_{k=0}^{n} P_{M_{n, k}}(t)-\sum_{k=0}^{n-1} Q_{E_{k, k+1}^{n}}(t)$ is the Poincaré polynomial of $C^{[n]}$. It is easy to see that $\sum_{n \geqslant 0} q^{n}\left(\sum_{k=0}^{n} P_{M_{n, k}}(t)-\sum_{k=0}^{n-1} Q_{E_{k, k+1}^{n}}(t)\right)=P_{V}(q, t)$.

Figure 1 shows the graded dimension of $V$ as a bigraded vector space.

\subsection{Computation of the A-action}

We will now investigate the action of the algebra $A=\left\langle x_{1}, x_{2}, \mu_{+}, \mu_{-}, d_{1}, d_{2}\right\rangle_{\mathbb{Q}}$ on $V$.

Consider $V_{\bullet}, 2 n=\bigoplus_{i} V_{i, 2 n} \subset V$, that is, all the classes in homological degree $2 n$. Denote by $\left[M_{n, k}\right]$ the fundamental class of the irreducible component $M_{n, k}$ of $C^{[n]}$ as described in the previous subsection.

Theorem 5.4. The fundamental classes $\left[M_{n, k}\right] \in V_{n, 2 n}$ generate $V_{\bullet, 2 n}$ as a $\mathbb{Q}\left[x_{1}, x_{2}\right]$-module. 


\begin{tabular}{|c|c|c|c|c|c|c|c|}
\hline & $\mathbf{0}$ & $\mathbf{2}$ & $\mathbf{4}$ & $\mathbf{6}$ & $\mathbf{8}$ & $\mathbf{1 0}$ & $\cdots$ \\
\hline $\mathbf{0}$ & 1 & 0 & 0 & 0 & 0 & 0 & $\cdots$ \\
\hline $\mathbf{1}$ & 1 & 2 & 0 & 0 & 0 & 0 & $\cdots$ \\
\hline $\mathbf{2}$ & 1 & 3 & 3 & 0 & 0 & 0 & $\cdots$ \\
\hline $\mathbf{3}$ & 1 & 4 & 5 & 4 & 0 & 0 & $\cdots$ \\
\hline $\mathbf{4}$ & 1 & 5 & 7 & 7 & 5 & 0 & $\cdots$ \\
\hline $\mathbf{5}$ & 1 & 6 & 9 & 10 & 9 & 6 & $\cdots$ \\
\hline$\vdots$ & $\vdots$ & $\vdots$ & $\vdots$ & $\vdots$ & $\vdots$ & $\vdots$ & $\ddots$ \\
\hline
\end{tabular}

Figure 1. The dimensions $V_{n, d}$, that is, the Betti numbers of $C^{[n]}$; the columns are labeled by homological degree $d$ and the rows by the number of points $n$

Proof. This is equivalent to proving that the maps $\left.x_{i}\right|_{V_{k, 2 n}}$ are jointly surjective for $k \geqslant n$. If we dualize the maps to pullbacks $\left.x_{i}^{*}\right|_{k, 2 n}$ in cohomology, this condition says that the operators $x_{i}^{*}: H^{<2 k+2}\left(C^{[k+1]}\right) \rightarrow H^{*}\left(C^{[k]}\right)$ must satisfy $\bigcap \operatorname{ker} x_{i}^{*}=0$.

We have the following diagram for the components of $C^{[n]}$ and their intersections:

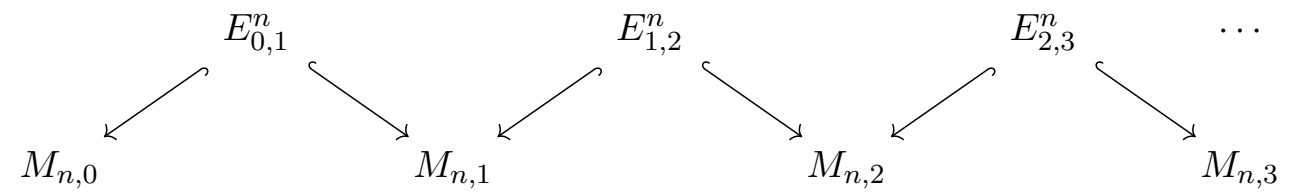

Since $C^{[n]}$ is a chain of the components $M_{k, n-k}$ intersecting transversally, without triple intersections, the Mayer-Vietoris sequence in homology for unions splits to short exact sequences:

$$
0 \rightarrow \bigoplus_{k=0}^{n-1} H_{i}\left(E_{k, k+1}^{n}\right) \rightarrow \bigoplus_{k=0}^{n} H_{i}\left(M_{n, k}\right) \rightarrow H_{i}\left(C^{[n]}\right) \rightarrow 0 .
$$

Dually, we have an exact sequence the other way around in cohomology. By our blowup description of $\pi: M_{n, k} \mapsto \mathbb{P}^{n-k} \times \mathbb{P}^{k}$, we have the following equality of graded vector spaces (see, for example, [GH78, Chapter 6]):

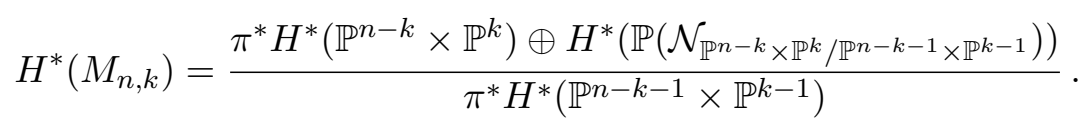

In particular, we can write

$$
H^{*}\left(\mathbb{P}^{n-k} \times \mathbb{P}^{k}\right)=\mathbb{Q}\left[a_{n, k}, b_{n, k}\right] /\left(a_{n, k}^{n-k+1}, b_{n, k}^{k+1}\right)
$$

as well as

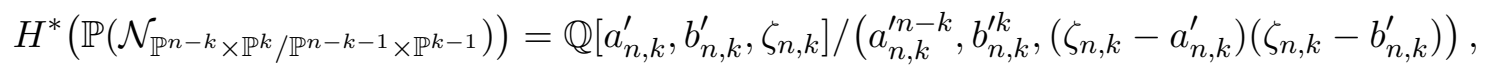

where $\zeta_{n, k}=c_{1}(\mathcal{O}(1))$.

Since the classes

$$
a_{n, k}^{\prime \prime i} b_{n, k}^{\prime \prime j} \in \pi^{*} H^{*}\left(\mathbb{P}^{n-k-1} \times \mathbb{P}^{k-1}\right) \cong \mathbb{Q}\left[a_{n, k}^{\prime \prime}, b_{n, k}^{\prime \prime}\right] /\left(a_{n, k}^{\prime \prime n-k}, b_{n, k}^{\prime \prime k}\right)
$$

are identified with $a_{n, k}^{i} b_{n, k}^{j}$ in $\pi^{*} H^{*}\left(\mathbb{P}^{n-k} \times \mathbb{P}^{k}\right)$, where $i<n-k$ and $j<k$, and with $a_{n, k}^{i} b_{n, k}^{\prime j}$ in the exceptional divisor, the quotient map as graded $\mathbb{Q}$-modules is the one identifying $a_{n, k}^{i} b_{n, k}^{j}$ 


\section{O. Kivinen}

with $a_{n, k}^{\prime i} b_{n, k}^{\prime j}$.

All in all, we can write that, as graded $\mathbb{Q}$-vector spaces,

$$
H^{*}\left(M_{n, k}\right)=\left\langle 1, a_{n, k}, b_{n, k}, \ldots, a_{n, k}^{n-k} b_{n, k}^{k}, \zeta_{n, k}, \ldots, \zeta_{n, k} a_{n, k}^{n-k-1} b^{k-1}\right\rangle_{\mathbb{Q}} .
$$

Example 5.5. We have $H^{*}\left(M_{2,0}\right)=\operatorname{span}\left\{1, a_{3,0}, a_{3,0}^{2}\right\}$, as expected, since $M_{2,0} \cong \mathbb{P}_{\mathbb{C}}^{2}$. We also have $H^{*}\left(M_{2,1}\right)=\operatorname{span}\left\{1, a_{2,1}, a_{2,1}^{2}, b_{2,1}, a_{2,1} b_{2,1}, a_{2,1}^{2} b_{2,1}, \zeta_{2,1}, \zeta_{2,1} a_{2,1}\right\}$.

Having described the cohomology of the components $M_{n, k}$, we can get back to our exact sequence. Identify

$$
H^{*}\left(\mathbb{P}^{n-k-1} \times \mathbb{P}^{k}\right) \cong \mathbb{Q}\left[\mu_{n, k}, \nu_{n, k}\right] /\left(\mu_{n, k}^{n-k}, \nu_{n, k}^{k+1}\right) .
$$

Lemma 5.6. Under the inclusion $E_{k, k+1}^{n} \hookrightarrow M_{n, k}$, we have $\mu_{n, k} \mapsto a_{n, k}-\zeta_{n, k}$ and $\nu_{n, k} \mapsto b_{n, k}-\zeta_{n, k}$ in cohomology. Similarly, under the inclusion $E_{k, k+1}^{n} \hookrightarrow M_{n, k+1}$, we have $\mu_{n, k} \mapsto a_{n, k+1}-\zeta_{n, k+1}$ and $\nu_{n, k} \mapsto b_{n, k+1}-\zeta_{n, k+1}$.

Proof. The class of $\mu_{n, k}$ in the intersection is the class dual to the line $L_{n, k}^{y}$, where we fix all points in $E_{k, k+1}^{n}$ at the origin except for one at the $y$-axis. Similarly, the class of $\nu_{n, k}$ is the line $L_{n, k}^{x}$, where we have but one point on the $x$-axis. Under the blowup $\pi_{n, k}: M_{n, k} \rightarrow \mathbb{P}^{n-k} \times \mathbb{P}^{k}$, the class of $L_{n, k}^{y}$ in $M_{n, k}$ is given by the total transform, which satisfies $\left[L_{n, k}^{y}\right]+\zeta_{n, k}=a_{n, k}$. The computation for the other three cases is nearly identical, and we omit it.

Example 5.7. When $n=2$, the Hilbert scheme $C^{[2]}$ has the following components: $M_{2,0} \cong M_{2,2} \cong$ $\mathbb{P}^{2}$ and $M_{2,1} \cong \mathrm{Bl}_{\mathrm{pt}}\left(\mathbb{P}^{1} \times \mathbb{P}^{1}\right)$. The intersections are $E_{0,1}^{2} \cong E_{1,2}^{2} \cong \mathbb{P}^{1}$. The fundamental class of the first intersection is denoted by $\mu_{2,0}$ and that of the second one is denoted by $\mu_{2,1}$. Under the inclusion $E_{0,1}^{2} \hookrightarrow M_{2,0}$, the class $\mu_{2,0}$ is identified with $a_{2,0}$, and under the inclusion $E_{0,1}^{2} \hookrightarrow M_{2,1}$, it is identified with $a_{2,1}-\zeta_{2,1}$. Similarly, under the inclusion $E_{1,2}^{2} \hookrightarrow M_{2,1}$, the class $\mu_{2,1}$ is identified with $a_{2,1}-\zeta_{2,1}$, whereas under the inclusion $E_{1,2}^{2} \hookrightarrow M_{2,2}$, it is identified with $a_{2,2}$.

As follows from the definition of the maps $\iota_{i}: C^{[n]} \rightarrow C^{[n+1]}$, we can consider them as restricted to $M_{n, k}$. They induce, by abuse of notation, maps in cohomology $x_{i}^{*}: H^{*}\left(M_{n+1, k+i-1}\right) \rightarrow$ $H^{*}\left(M_{n, k}\right)$. We can describe these maps explicitly.

LEMma 5.8. In the basis of (5.1), the map $x_{1}^{*}$ is

$$
a_{n+1, k}^{i} b_{n+1, k}^{j} \mapsto a_{n, k}^{i} b_{n, k}^{j}, \quad \zeta_{n+1, k} a_{n+1, k}^{i} b_{n+1, k}^{j} \mapsto \zeta_{n, k} a_{n, k}^{i} b_{n, k}^{j} .
$$

Similarly, the map $x_{2}^{*}$ is

$$
a_{n+1, k+1}^{i} b_{n+1, k+1}^{j} \mapsto a_{n, k}^{i} b_{n, k}^{j}, \quad \zeta_{n+1, k+1} a_{n+1, k+1}^{i} b_{n+1, k+1}^{j} \mapsto \zeta_{n, k} a_{n, k}^{i} b_{n, k}^{j} .
$$

Proof. We are adding one fixed smooth point, that is, an embedding $C^{[n]} \hookrightarrow C^{[n+1]}$, as a divisor. When we blow down the components, it is immediate that the $a$-classes go to the $a$-classes and the $b$-classes go to the $b$-classes. We can treat the classes in the exceptional divisor separately; there, everything again reduces to embedding products of projective spaces as above. In addition, we need that $x_{1}^{*} \zeta_{n+1, k+1}=\zeta_{n, k}$, which is saying that the normal bundle of the exceptional divisor of $M_{n+1, k+1}$ restricts to that of the exceptional divisor of $M_{n, k}$ under the embedding $\iota_{1}: M_{n, k} \rightarrow M_{n+1, k+1}$. In the notation of Lemma 5.1, on $M_{n, k}$, the map $\iota_{1}$ is given by multiplying $a(x)$ by $x-c$ for some fixed $c \neq 0$. In particular, the centers of the blowups become identified, and the restriction of the normal bundle of the exceptional divisor of $M_{n+1, k+1}$ is the normal bundle of $M_{n, k}$. 


\section{Homology of Hilbert SCHEMES OF PLANAR CURVES}

Having the above lemmas at our hands, we want to prove that the intersections of the kernels of the $x_{i}^{*}$ are only the fundamental classes.

The basic object of study here is the commutative diagram

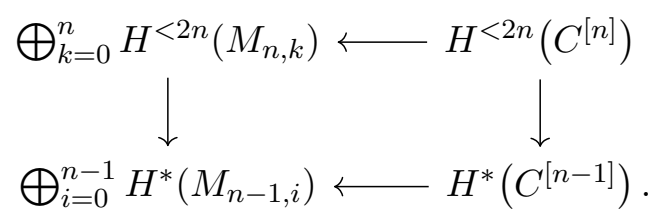

We can explicitly describe the kernels on the left: for each $M_{n, k}$, only the classes $\zeta_{n, k} a_{n, k}^{k-1} b_{n, k}^{n-k-1}$ are in their intersection. In particular, the intersection of the kernels is nonempty. But this can be remedied on the right, as follows. By Lemma 5.6 and the Mayer-Vietoris sequence, inside the intersection, we can check that

$$
\begin{aligned}
x_{2}^{*}\left(\sum_{k} \lambda_{k} \zeta_{n, k} a_{n, k}^{k-1} b_{n, k}^{n-k-1}\right) & =\sum_{k} \lambda_{k} x_{2}^{*}\left(\zeta_{n, k}\right) a_{n-1, k}^{k-1} b_{n-1, k}^{n-k-1} \\
& =\sum_{k} \lambda_{k} x_{2}^{*}\left(a_{n, k}-a_{n, k+1}+\zeta_{n, k+1}\right) a_{n-1, k}^{k-1} b_{n-1, k}^{n-k-1} \\
& =\sum_{k} \lambda_{k}\left(a_{n-1, k-1}-a_{n-1, k}\right) a_{n-1, k}^{k-1} b_{n-1, k}^{n-k-1} \\
& =\sum_{k} \lambda_{k} a_{n-1, k-1} a_{n-1, k}^{k-1} b_{n-1, k}^{n-k-1},
\end{aligned}
$$

which is 0 if and only if $\lambda_{k}=0$ for all $k$. Repeating this for $x_{1}^{*}$, we have

$$
x_{1}^{*}\left(\sum_{k} \lambda_{k} \zeta_{n, k} a_{n, k}^{k-1} b_{n, k}^{n-k-1}\right)=\sum_{k} \lambda_{k} b_{n-1, k-1} a_{n-1, k}^{k-1} b_{n-1, k}^{n-k-1}=0
$$

if and only if $\lambda_{k}=0$ for all $k$. In particular, we see that the image of the fundamental class of the exceptional divisor is also nonzero; that is, it is not in the kernel and $\bigcap_{i} \operatorname{ker} x_{i}^{*}=0$ on the right. This finishes the proof of Theorem 5.4.

Having Theorem 5.4 at our hands, we can finally restate Theorem 1.4.

TheOrem 5.9. Consider the bigraded vector space $V^{\prime \prime}=\mathbb{Q}\left[x_{1}, x_{2}, y_{1}, y_{2}\right]$ with $x_{i}$ in degree $(1,0)$ and $y_{i}$ in degree $(1,2)$. Consider the action of $A^{\prime}=\mathbb{Q}\left\langle x_{i}, \partial_{y_{i}}, \sum y_{i}, \sum \partial_{x_{i}}\right\rangle$ on this space as differential operators, and let $U$ be the submodule $\mathbb{Q}\left[x_{1}, x_{2}, y_{1}+y_{2}\right]\left(x_{1}-x_{2}\right)$. Define $V^{\prime}=V^{\prime \prime} / U$. Then $V \cong V^{\prime}$ as $A$-modules, where $A=\mathbb{Q}\left[x_{1}, x_{2}, d_{1}, d_{2}, \mu_{+}, \mu_{-}\right] \cong A^{\prime}$.

Proof. That $A^{\prime}$ is isomorphic to $A$ in this case follows from the commutation relations when we map $x_{i} \mapsto x_{i}, \partial_{y_{i}} \mapsto d_{i}$ and $\sum y_{i} \mapsto \mu_{+}, \sum \partial_{x_{i}} \mapsto \mu_{-}$. We can identify $V^{\prime}$ and $V$ as $A$-modules by letting the monomial $y_{1}^{i} y_{2}^{j} / i ! j$ ! correspond to the fundamental class of $M_{i+j, i}$. It is then clear that on the diagonal $\bigoplus_{n \geqslant 0} V_{n, 2 n}$, the operators $d_{1}, d_{2}, \mu_{+}, \mu_{-}$act as the corresponding differential operators in $A^{\prime}$. Namely, the Gysin maps $d_{1}$ and $d_{2}$ are given by intersection, from which it follows that $d_{1}\left[M_{i+j, i}\right]=\left[M_{i+j-1, i-1}\right]$ and $d_{2}\left[M_{i+j, i}\right]=\left[M_{i+j-1, i}\right]$. This can be compared to the fact that, for example, $\partial_{y_{1}} y_{1}^{i} y_{2}^{j} / i ! j !=y_{1}^{i-1} y_{2}^{j} /(i-1) ! j !$.

By the commutation relations, $\left[d_{1}^{i+1}, \mu_{+}\right]=(i+1) d_{1}^{i}$, so

$$
\left[d_{1}^{i+1}, \mu_{+}\right] y_{1}^{i} y_{2}^{j} / i ! j !=(i+1) y_{2}^{j} / j !, \quad\left[d_{2}^{j+1}, \mu_{+}\right] y_{1}^{i} y_{2}^{j} / i ! j !=(j+1) y_{1}^{i} / i !
$$




\section{O. Kivinen}

and, in particular,

$$
d_{1}^{i+1} \mu_{+} y_{1}^{i} y_{2}^{j} / i ! j !=(i+1) y_{2}^{j} / j !, \quad d_{2}^{j+1} \mu_{+} y_{1}^{i} y_{2}^{j} / i ! j !=(j+1) y_{1}^{i} / i ! .
$$

Since $\mu_{+} y_{1}^{i} y_{2}^{j} / i ! j !=\sum_{k=0}^{i+j+1} c_{k} y_{1}^{k} y_{2}^{i+j+1-k}$ for some constants $c_{k}$, we must have $c_{k}=0$ unless $k=i$ or $k=i+1$, in which case we have $c_{k}=1 / i ! j$ !. This shows that $\mu_{+}$can be identified with multiplication by $y_{1}+y_{2}$ on the diagonal; the same holds below the diagonal since $\mu_{+}$commutes with the action of $x_{1}$ and $x_{2}$. On the diagonal, the operator $\mu_{-}$acts as zero by degree reasons. An argument similar to the above shows that below the diagonal, $\mu_{-}$acts by $\partial_{x_{1}}+\partial_{x_{2}}$.

Since the maps $x_{i}$ are jointly surjective on the rows, by Theorem 5.4, we get a surjection $\phi: \mathbb{Q}\left[x_{1}, x_{2}, y_{1}, y_{2}\right] \rightarrow V$. This is an $A$-module homomorphism, by the above. Its kernel contains $U$ since $\left(x_{1}-x_{2}\right) \cdot 1=0$ and the actions of $x_{i}$ and $\mu_{+}$commute with the $x_{i}$.

Then, consider the graded dimensions/Poincaré series of $V^{\prime}$ and $V$. We have

$$
P_{V^{\prime}}(q, t)=P_{V^{\prime \prime}}(q, t)-P_{U}(q, t)=\frac{1}{(1-q)^{2}\left(1-q t^{2}\right)}-\frac{q\left(1-q t^{2}\right)}{(1-q)^{2}\left(1-q t^{2}\right)^{2}}=P_{V}(q, t),
$$

and since $\operatorname{ker} \phi \supseteq U$, we must have $\operatorname{ker} \phi=U$.

\section{ACKNOWLEDGEMENTS}

I would like to thank my advisor Eugene Gorsky for his keen interest in this work and his continuing support, as well as for suggesting to study homological correspondences on Hilbert schemes. In addition, I thank Eric Babson, Brian Osserman, Vivek Shende and Zhiwei Yun for helpful discussions.

\section{REFERENCES}

ACG11 E. Arbarello, M. Cornalba and P. A. Griffiths, Geometry of algebraic curves. Vol. II, Grundlehren math. Wiss., vol. 268 (Springer, Heidelberg, 2011); doi:10.1007/978-3-54069392-5.

Che98 J. Cheah, Cellular decompositions for nested Hilbert schemes of points, Pacific J. Math. 183 (1998), no. 1, 39-90; doi:10.2140/pjm.1998.183.39.

EH16 B. Elias and M. Hogancamp, On the computation of torus link homology, 2016, arXiv:1603. 00407.

FM81 W. Fulton and R. MacPherson, Categorical framework for the study of singular spaces, Mem. Amer. Math. Soc. 31 (1981), no. 243; doi:10.1090/memo/0243.

GH78 P. Griffiths and J. Harris, Principles of algebraic geometry, Pure Appl. Math. (Wiley-Interscience, New York, 1978).

GLS07 G.-M. Greuel, C. Lossen and E. Shustin, Introduction to singularities and deformations, Springer Monog. Math. (Springer, Berlin, 2007); doi:10.1007/3-540-28419-2.

GORS14 E. Gorsky, A. Oblomkov, J. Rasmussen and V. Shende, Torus knots and the rational DAHA, Duke Math. J. 163 (2014), no. 14, 2709-2794; doi:10.1215/00127094-2827126.

Gro96 I. Grojnowski, Instantons and affine algebras. I. The Hilbert scheme and vertex operators, Math. Res. Lett. 3 (1996), no. 2, 275-291; doi:10.4310/MRL.1996.v3.n2.a12.

Gro95 A. Grothendieck, Techniques de construction et théorèmes d'existence en géométrie algébrique IV: les schémas de Hilbert, Sémin. Bourbaki (1960/1961), Exp. No. 221, Astérisque 6 (Soc. Math. France, Paris, 1961), 249-276.

Hog17 M. Hogancamp, Khovanov-Rozansky homology and higher Catalan sequences, 2017, arXiv: 1704.01562. 


\section{Homology of Hilbert SCHEMES OF PLANAR CURVES}

KR08 M. Khovanov and L. Rozansky, Matrix factorizations and link homology. II, Geom. Topol. 12 (2008), no. 3, 1387-1425; doi:10.2140/gt.2008.12.1387.

KR16 - Positive half of the Witt algebra acts on triply graded link homology, Quantum Topol. 7 (2016), no. 4, 737-795; doi:10.4171/QT/84.

Mel16 A. Mellit, Toric braids and (m,n)-parking functions, 2016, arXiv:1604.07456.

MRV17 M. Melo, A. Rapagnetta and F. Viviani, Fine compactified Jacobians of reduced curves, Trans. Amer. Math. Soc. 369 (2017), no. 8, 5341-5402; doi:10.1090/tran/6823.

MS13 L. Migliorini and V. Shende, A support theorem for Hilbert schemes of planar curves, J. Eur. Math. Soc. 15 (2013), no. 6, 2353-2367; doi:10.4171/JEMS/423.

MSV18 L. Migliorini, V. Shende and F. Viviani, A support theorem for Hilbert schemes of planar curves. II, 2018, arXiv:1508.07602.

MY14 D. Maulik and Z. Yun, Macdonald formula for curves with planar singularities, J. reine angew. Math. 694 (2014), 27-48; doi:10.1515/crelle-2012-0093.

Nak97 H. Nakajima, Heisenberg algebra and Hilbert schemes of points on projective surfaces, Ann. of Math. 145 (1997), no. 2, 379-388; doi:10.2307/2951818.

Ngô06 B. C. Ngô, Fibration de Hitchin et endoscopie, Invent. Math. 164 (2006), no. 2, 399-453; doi: 10.1007/s00222-005-0483-7.

ORS18 A. Oblomkov, J. Rasmussen and V. Shende, The Hilbert scheme of a plane curve singularity and the HOMFLY homology of its link, Geom. Topol. 22 (2018), no. 2, 645-691; doi:10.2140/ gt.2018.22.645.

OY16 A. Oblomkov and Z. Yun, Geometric representations of graded and rational Cherednik algebras, Adv. Math. 292 (2016), 601-706; doi:10.1016/j.aim.2016.01.015.

Ran05a Z. Ran, Geometry on nodal curves, Compos. Math. 141 (2005), no. 5, 1191-1212; doi:10. 1112/S0010437X05001466.

Ran05b_, A note on Hilbert schemes of nodal curves, J. Algebra 292 (2005), no. 2, 429-446; doi:10.1016/j.jalgebra.2005.06.028.

Ras15 J. Rasmussen, Some differentials on Khovanov-Rozansky homology, Geom. Topol. 19 (2015), no. 6, 3031-3104; doi:10.2140/gt.2015.19.3031.

Ren18 J. V. Rennemo, Homology of Hilbert schemes of points on a locally planar curve, J. Eur. Math. Soc. 20 (2018), no. 7, 1629-1654; doi:10.4171/JEMS/795.

Ser06 E. Sernesi, Deformations of algebraic schemes, Grundlehren math. Wiss., vol. 334 (SpringerVerlag, Berlin, 2006); doi:10.1007/978-3-540-30615-3.

She12 V. Shende, Hilbert schemes of points on a locally planar curve and the Severi strata of its versal deformation, Compos. Math. 148 (2012), no. 2, 531-547; doi:10.1112/S0010437X11007378.

Tik97 A.S. Tikhomirov, The variety of complete pairs of zero-dimensional subschemes of an algebraic surface, Izv. Math. 61 (1997), no. 6, 1265-1291; doi:10.1070/im1997v061n06ABEH000169.

VV09 M. Varagnolo and E. Vasserot, Finite-dimensional representations of DAHA and affine Springer fibers: the spherical case, Duke Math. J. 147 (2009), no. 3, 439-540; doi:10.1215/ 00127094-2009-016.

Oscar Kivinen okivinen@ucdavis.edu

Department of Mathematics, University of California, Davis, One Shields Avenue, 95616 Davis, CA, USA 